\title{
Geographische Fachlichkeit - didaktische Erschließung durch Basiskonzepte
}

\author{
*kerstinandrea@hotmail.com \\ “* peterca@staff.uni-marburg.de, Phillipps Universität Marburg, ( $\square$ korresp. Autorin) \\ ***nauss@staff.uni-marburg.de,Phillipps Universität Marburg \\ eingereicht am: 09.03.2020, akzeptiert am: 07.01.2021
}

\begin{abstract}
Die Fertigkeit komplexe Probleme und Situationen unter sozialwissenschaftlicher und naturwissenschaftlicher Perspektive - in einer Mensch-Umwelt-Schnittstelle - zu erfassen und zu verstehen, ist im Hinblick auf eine adäquate fachliche Vermittlung im Geographieunterricht zentral und kann eine Herausforderung, insbesondere für angehende Lehrer*innen darstellen. Um dieser zu begegnen, wurde ein geographisches Erschließungsmodell (GEM), welches die Identifizierung von fachlichen Zugängen ermöglichen soll und vor der didaktischen Analyse ansetzt, entwickelt. Es kann als Hinführung zur Sachanalyse angewendet werden und basiert auf drei Basiskonzepten (space, place, scale). Ziel ist die Strukturierung von Problemen und Situationen, also von Fachgegenständen, die durch eine fachdidaktische Herangehensweise ermöglicht werden soll. Dabei sollen die den Fachgegenständen zugrunde liegende Komplexität erkannt und unter unterschiedlichen Perspektiven jeweils dekonstruiert werden. In diesem Artikel wird GEM am Beispiel des Brandrodungswanderfeldbaus und dem ProblembeispielBevölkerungsdruck/Raubbau im Tropischen Regenwald veranschaulicht.
\end{abstract}

Keywords: Fachlichkeit, Basiskonzepte, Mensch/Umwelt, Lehrerprofessionalisierung

\section{Geographical expertise - didactic comprehension through key concepts}

The ability to grasp and understand complex problems and situations under a social science and scientific perspective linked to a ,human-environment interface ${ }^{\text {c }}$ - is central regarding to an adequate specialist teaching in geography lessons and can be a challenge for (prospective) teacher. In order to meet this challenge, a geographical comprehension model (GEM) was developed, which is designed for the identification of subject related approaches and which starts before the didactic analysis. It can be used as an introduction to a subject analysis and is based on three basic key concepts (space, place, scale). The aim is to structure problems and situations, i.e. topics, with a subject-didactic approach. In doing so, the underlying complexity of the topics should be recognised and made comprehensible from different perspectives. In this article, $G E M$ is realized on the topic of "slash-and-burn shifting cultivation" and the example of "population pressure/overexploitation in the tropical rainforest".

Keywords: disciplinary knowledge, key concepts, human/environment, teacher professionalization

\section{Einführung}

Das Unterrichtsfach Geographie zeichnet sich durch seine Auseinandersetzung mit Systemen im Kontext der Mensch-Umwelt-Schnittstelle aus. Die in der Schule zu behandelnden Probleme und Situationen sollen von den Studierenden und (angehenden) Lehrkräften im Hinblick auf den Schulunterricht fachlich und didaktisch erschlossen und vorbereitet werden. Die Frage, wie Inhalte fachlich adäquat bearbeitet wer- den können, ist von besonderem Interesse, weil die zu unterrichtenden Probleme und Situationen sich oftmals durch eine hohe Komplexität auszeichnen. Die Komplexität zeigt sich bspw. in einer „,Vielfalt und Vernetzung zahlreicher Einflussgrößen“(Ohl 2013: 5).

In Vorbereitung auf einen kompetenzorientierten Schulunterricht sollte ein tiefes Verständnis für die Fachgegenstände entwickelt werden, auch wenn die Gesamtheit der fachwissenschaftlichen Inhalte in Bezug darauf für den Geographieunterricht didaktisch 
konstruiert werden. Um Inhalte in Hinblick auf die Alltagswelt unterrichten zu können, ist es unerlässlich, dass für den Erwerb und die Förderung von Kompetenzen, die Fachgegenstände aus mehreren Perspektiven inhaltlich durchdrungen werden. Die Studie COACTIV (vgl. Krauss et al. 2008; Kunter et al. 2011) stellte aus mathematischer Perspektive bereits deutlich die Notwendigkeit eines fundierten Fachwissens dar. Es wurde dargelegt, dass ein „fundiertes Fachwissen“ gewährleistet, ,,dass Argumentationsweisen und das Herstellen von Zusammenhängen, [...] derart erfolgen kann, dass es an die typischen Wissensbildungsprozesse des Faches [...] anschließen kann“; Fachwissen sollte demnach ein ,tieferes Verständnis der Inhalte“ (Krauss et al. 2008: 237f.) einbeziehen. Es ist aber auch klar, dass rein fachliches Wissen einer Lehrperson nicht für einen gelungenen Unterricht ausreicht.

Fachlichkeit weist immer eine didaktische Sichtweise auf, indem die „didaktische Rekonstruktion fachlichen Wissens [...] auf das Lehren und Lernen und die subjektive Aneignung ausgerichtet" (Hericks \& Laging 2020: 123 f.) wird. Für den Geographieunterricht bedeutet das, dass vor dem Schritt der eigentlichen Unterrichtsplanung die Lehrpersonen fähig sein sollten, den Fachgegenstand in seiner Struktur zu durchdringen. Passend hierzu stellt Shulman (2004: 235) dar, dass bevor man Inhalte unterrichtet, diese zunächst verstanden werden müssen: „To teach is first to understand". Aufgrund der Komplexität der Fachgegenstände sollten Geographiestudierende erlernen, Komplexität adäquat zu dekonstruieren und Inhalte zu strukturieren. Ferner sollten sie ihr Allgemein-/Alltagswissen, ihre Vorurteile und Überzeugungen über die Inhalte fachlich reflektieren. Studien zeigen weit verbreitete Defizite bei Geographielehramtsstudierenden im Verständnis der wissenschaftlichen Konzepte, die dem geographischen Wissen zugrunde liegen (vgl. Arslan et al. 2012; Khalid 2003; Reinfried 2006). Hericks und Meister (2020) formulieren die Kernidee des doppelten Praxisverständnisses, in welcher das Fachwissen und das Fachverständnis eine zentrale Rolle für das Gymnasiallehrer*innenstudium spielen. Im Hinblick auf die Lehramtsausbildung geht es hierbei um die didaktische Reflexion des fachlichen Wissens. Damit der fachdidaktische Kern erkannt wird, ist umfassendes Fachverständnis unerlässlich (ebd.). Die erste Praxis umfasst dabei die eigene wissenschaftliche Auseinandersetzung mit Lerngegenständen und Themen, in der Verstehensprobleme reflektiert werden; die zweite Praxis stellt die Unterrichtstätigkeit im Rahmen von Schulpraktika dar (vgl. Hericks et al. 2018; Hericks \& Laging 2020).

Eine didaktische Sichtweise auf das fachliche Wissen ist also notwendig. Ohnehin muss an die- ser Stelle nicht gesagt werden, dass Fachwissen und fachdidaktisches Wissen nur schwer voneinander abgegrenzt werden können und oft miteinander verschmelzen. Fachwissenschaftliches und fachdidaktisches Wissen sollen zum Können verschmelzen (vgl. Neuweg 2014).

In diesem Artikel wird der Frage nachgegangen, wie Studierenden dabei unterstützt werden können. Konkret geht es darum, wie Fachgegenstände Geographielehramtsstudierenden zugänglich gemacht werden können. Unser Ziel ist ein geographisches Erschließungsmodell (GEM), das zur Inhaltskonstruktion genutzt werden kann und einen fachlich geleiteten Zugang zum Geographieunterricht ermöglicht. Basiskonzepte dienen dabei als theoretische Grundlage für die Entwicklung des GEMs, da postuliert wird, dass deren Anwendung Lehrkräfte bei der Strukturierung von Inhalten unterstützen wie auch geographisches Denken begünstigen können (vgl. DGfG 2020; Lambert 2013; Uhlenwinkel 2013a, 2013b).

Die Fragen, die in der vorliegenden Arbeit im Vordergrund stehen, sind:

- Welche Basiskonzepte gibt es und für welchen Zweck werden diese verwendet?

- Welche Basiskonzepte und Methoden der Disziplin könnten verwendet werden, um einen Fachgegenstand zu erschließen?

- Wie könnte ein fachdidaktisches Erschließungsmodell aussehen, welches mithilfe von spezifischen Basiskonzepten bei der Erschließung eines Fachgegenstands methodische Unterstützung liefert?

Unsere gesammelten Erfahrungen in Rahmen von Seminaren, in denen Lehramtsstudierende sich mit curricularen Thematiken auseinandersetzten und Unterricht planen sollten, zeigten, dass es den Studierenden teilweise schwer fiel, die vorgegebenen Probleme und Situationen in ihrer Struktur zu erschließen.

Es kann angenommen werden, dass bestimmte Basiskonzepte Studierenden und Lehrkräften auch bei dem Erschließungsvorgang zum fachdidaktischen Wissen helfen können. Da allerdings von den Autor*innen unterschiedliche Basiskonzepte ausgewählt und verwendet werden, wurde zunächst eine Literaturrecherche mit anschließender Analyse der geographischen Konzepte und Basiskonzepte auf internationaler Ebene durchgeführt. Hierbei ging es vor allem um die Ziele und Anwendungen der Konzepte sowie einer Eruierung, welche Konzepte verstärkt vertreten sind.

- Im Hinblick auf die Professionalisierung von Lehramtsstudierenden wurden daraus Konzepte abgeleitet und formuliert, die bei der fachdidaktischen Erschließung von Fachgegenständen helfen können. 
- Basierend auf dem verfolgten Ziel wurde ein geographisches Erschließungsmodell entwickelt. Auswahl und Begründung der Basiskonzepte für das GEM wie auch das GEM selbst, werden in diesem Artikel beschrieben.

\section{Theoretischer Hintergrund}

\subsection{Geographie in Schule und Hochschule}

Fachgegenstände und deren Inhalte sind aufgrund ihrer zeit- und maßstabsübergreifenden Multidimensionalität sehr komplex. Da Studierende im Studium, im Fachpraktikum, im Referendariat und dann vor allem als Lehrkraft mit diesen umgehen müssen, sollte der fachliche Umgang bereits frühzeitig an Hochschulen reflektiert und trainiert werden.

An dieser Stelle soll zunächst Bezug auf die Struktur des Faches genommen werden: An den Universitäten in Deutschland besteht Geographie quasi aus einer Zwei-Fächer-Disziplin, die durch eine hohe Spezialisierung der Forschung im Bereich der physischen Geographie und der Humangeographie gekennzeichnet ist (vgl. Clifford 2002; Thrift 2002; Weichhart 2005). Neben der sensiblen Wahrnehmung der räumlichen Relativität von physisch-materiellen Dingen, Körpern und Phänomenen (vgl. Massey 1999; Weichhart 1999; Weichhart 2005) haben die beiden Unterdisziplinen nicht mehr viel gemeinsam.

Aufgrund der fehlenden Einheit der Humangeographie und der physischen Geographie postuliert Weichhart (2005) in seinem 3-Säulen-Modell, neben der physischen und Humangeographie, eine dritte Teildisziplin, die auf einer sozial-ökologischen Forschung basiert. Dabei könnten drei Unterdisziplinen existieren und die Humangeographie sowie die physische Geographie würden ihre Spezialisierung nicht verlieren. Darüber hinaus zeigt die Delphi-Studie (vgl. Weichhart 2005) Probleme einer möglichen gemeinsamen Forschung im Kontext von Mensch-UmweltInteraktionen auf. So arbeiten die meisten Kooperationsprojekte wohl mehr für sich als gemeinsam (ebd.). Es bestehen Bemühungen, das Anthropozän als geologische Epoche zu etablieren, um den Einflussfaktor Mensch auf den Planeten in den Fokus zu rücken. Dies befördert ferner die Debatte, die Humangeographie und physische Geographie zu vereinen (vgl. Geist 2018).

Das Schulfach wird verstärkt als interdisziplinäre Brücke zwischen Natur- und Gesellschaftswissenschaften gesehen (vgl. DGfG 2020) und begründet somit das Zusammenhalten der physischen Geographie und der Humangeographie in Deutschland (vgl. Wardenga 2011).

\subsection{Geographie - Lehramt an Hochschulen}

Die erlebte Freude am Schulfach spielt eine entscheidende Rolle bei der Studienfachwahl an Universitäten (vgl. Steinbrick \& Aufenvenne 2016). Die Studierenden verfügen über eine bestimmte Vorstellung bezüglich ihres Wahlfaches und kommen mit dieser an die Universität. Dort stellen zukünftige Lehrpersonen jedoch eine Diskrepanz fest zwischen dem, was sie in der Schule gelernt und gelehrt bekommen haben, und dem, was an der Universität diskutiert wird (vgl. Wardenga 2011). Der Grund dafür ist die zunehmende Differenzierung der Geographie an der Forschungsfront, wodurch die Verbindung zwischen Hochschulgeographie und Schulgeographie geschwächt wird (vgl. Clifford 2002), und die curricularen Themen, die für die späteren Lehrkräfte im Berufsleben relevant sein werden, werden an der Universität selten behandelt (vgl. Hemmer \& Hemmer 2000; Hemmer \& Obermeier 2003).

Weder die Humangeographie noch die physische Geographie kann die bestehende Lücke zwischen Menschen und Umwelt in der Hochschulbildung mit dem in der Schule praktizierten Ansatz füllen. Somit ist ein Defizit der Lehrerprofessionalisierung an Universitäten von Beginn der Qualifikation an zu beobachten. Ein Beitrag zur Behebung des Defizits soll mit dem GEM zur Diskussion gestellt werden. Indem $G E M$ als Brückenfachstrategie gesehen wird, werden sowohl Zugänge einer sozialwissenschaftlichen wie auch einer naturwissenschaftlichen Geographie auf bestimmte Fachgegenstände ermöglicht.

\subsection{Geographische Basiskonzepte}

Basiskonzepte sind fachspezifische Konstrukte, die jedoch innerhalb der geographischen Disziplin variieren. ,At a basic level, concepts are classifiers - ways of dividing up our experience of a complex and messy world into more manageable units, enabling us to communicate about things we can't immediately see" (Taylor 2008: 50). Chancen der Konzepte für den Geographieunterricht werden in Deutschland, Österreich, Großbritannien, Australien, etc. gesehen, wobei es sich aber um keine fest definierten Begrifflichkeiten handelt (vgl. Lambert \& Morgan 2010). Basiskonzepte wurden und werden im Hinblick auf ein didaktisches Ziel konzipiert und können sich daher von Land zu Land unterscheiden, je nachdem, welches Bildungsziel das geographische Fach verfolgt. So bilden in der deutschen Schulgeographie MenschUmwelt-Beziehungen die Leitidee des Faches ab. Die USA formulieren bspw. neben 'earth's place in the universe' und 'earth's system', 'earth and human activity' als disziplinäre Kernideen im Rahmen des K-12 Science Education Framework (vgl. National Research 
Council 2012). Darüber hinaus wird Geographie durch zwei Perspektiven definiert: räumlich und ökologisch (vgl. Heffron \& Downs 2012). Im nationalen Curriculum Großbritanniens spielen auch physische und humangeographische Prozesse eine wichtige Rolle (vgl. National Curriculum in England 2013). Dasselbe gilt auch für Australien, wo ,,deep knowledge and understanding of why the world is the way it is and the interconnections between people, places and environments over place and time" (vgl. australischer Lehrplan 2014) erforderlich sind. Basiskonzepte tauchten zuerst im englischsprachigen Raum auf und werden oft explizit im Kontext der geographischen Bildung genannt, z. B. als Ziel geographisches Wissen und Verständnis zu beschreiben und zu analysieren (vgl. Brooks 2013).

Die Bildungsstandards in Deutschland beinhalten das Systemkonzept als Kern, welches sich auf die Teilkonzepte Struktur, Funktion, Prozess als Systemkomponenten bezieht. Die zentrale Untersuchung besteht aus den Zusammenhängen zwischen physikalischen und geographischen Subsystemen. So sollen die Konzepte bspw. Lehrkräfte unterstützen, indem sie die Inhalte strukturieren und den Lernenden helfen, spezifische Strukturen, Funktionen und Prozesse in verschiedenen Räumen und Beziehungen während des gesamten Unterrichts in der Schule zu erkennen und mit anderen Fächern in der Schule zu verbinden (vgl. DGfG 2020). Das Problem dieser Konzeption besteht in der Überschneidung von fachspezifischen Basiskonzepten verschiedener Schulfächer. So werden beispielsweise auch in der Biologie die Konzepte Struktur und Funktion verwendet (KMK 2004). Somit sind es vordergründig keine rein fachlichen Basiskonzepte.

Fögele (2016) analysierte die Rekonstruktion von Lehrkräfteorientierungen basierend auf konzeptbasiertem Geographieunterricht im Rahmen einer Lehrkräftefortbildung und zeigte die Schwierigkeit auf, die bestehenden Überzeugungen der Lehrkräfte weiterzuentwickeln. Mehren (2013: 22) beschreibt den Einsatz von geographischen Basiskonzepten in der deutschen Bildung als ,Erklärungsansätze und Leitideen des fachlichen Denkens, die sich in unterschiedlichen geographischen Sachverhalten wiederfinden lassen" und für Lehrkräfte als Instrument der Unterrichtsplanung dienen (vgl. Uphues 2013). Zudem können Basiskonzepte zur fachlichen Analyse von Inhalten genutzt werden (vgl. Fögele 2015).

Auch Ammoneit et al. (2019) verwenden für ihr geographisches Kompetenzmodell im Zuge der Modellierung von Raum, die in der DGfG (2020) vorgestellten Basiskonzepte Struktur, Funktion und Prozess als hierarchische Abstraktionsebenen. Die Anwendung des Kompetenzmodells zielt darauf $a b$, eine zweckgerichtete Modellierung räumlich-komplexer Kontexte zu ermöglichen (ebd.).
Auch in dem österreichischen GW- Lehrplan AHS Sek II sind Basiskonzepte fest integriert und dienen Lehrkräften bei der Unterrichtsplanung und -gestaltung aber auch Lernenden als „Orientierungshilfe [...], eine komplexe Welt lesbar, verhandelbar und gestaltbar" (Jekel \& Pichler 2017: 8) zu erleben. Sie können zur ,Analyse und Reflexion neuer fachlicher Kontexte dienen und in vergleichbaren Feldern der Lebenswelt anwendbar sein" (ebd.). Hier sind $13 \mathrm{Ba}-$ siskonzepte ${ }^{1}$ formuliert, welche bewusst Ähnlichkeiten mit Basiskonzepten anderer Länder aufweisen.

Lambert (2007) schlägt vor, dass Konzepte wie space/place/interconnectedness in Bezug auf die didaktische Ausbildung verwendet werden, um ein Verständnis zu erlangen, wie soziale, ökologische, wirtschaftliche und politische Prozesse zusammenhängen. Dabei stellen sie keinen neuen Inhalt dar, sondern eher die Architektur, die dem Fach seine Struktur gibt und seinen Zweck bekräftigt.

Das 2007 vorgestellte Geography National Curriculum für Großbritannien benannte sieben Basiskonzepte (place, space, scale, environmental interaction and sustainable development, physical and human processes, interdependence, cultural understanding and diversity), die als hierarchisch zu verstehen sind, da sie helfen sollten, die Inhalte und Ideen des Faches in Form eines Content Containers, basierend auf place, space und scale, zu gruppieren (Brooks 2013: $76 \mathrm{f}$.). Dieser Ansatz zielte zum einen darauf ab, Lehrpersonen bei der Planung und Entwicklung des Curriculums zu unterstützen, und zum anderen, Lernenden bei der schrittweisen Entwicklung über das Verständnis der Konzepte zu helfen, indem diese die Orte und Themen untersuchen sollten, die die Lehrkraft für sie ausgewählt hat (vgl. Rawling 2007). Brooks (2013) stellt jedoch dar, dass es keinen konkreten Konsens darüber gab, ob diese Konzepte tatsächlich eine Basis für Geographieunterricht darstellten oder ob es sich hierbei ausschließlich um geographische Konzepte handelte. Ein weiteres Beispiel mit sieben Konzepten stellt Taylor $(2008,2011)$ vor: Die hierarchischen Konzepte: place, space und time, und die vier Organisationskonzepte: diversity, interaction, change und perception and representation, die helfen, Inhalte, die gelehrt werden sollen, zu klassifizieren (Planung) und diese Inhalte durch Leitfragen zu strukturieren. Die Schüler*innen sollen dann die im Hinblick auf die Organisationskonzepte formulierten Leitfragen in Unterrichtseinheiten bearbeiten. Ferner helfen die

\footnotetext{
Raumkonstruktion und Raumkonzepte; Regionalisierung und Zonierung; Diversität und Disparität; Maßstäblichkeit; Wahrnehmung und Darstellung; Nachhaltigkeit und Lebensqualität; Interessen, Konflikte und Macht; Arbeit, Produktion und Konsum; Märkte, Regulierung und Deregulierung; Wachstum und Krise; Mensch-Umwelt Beziehungen; Geö̈kosysteme; Kontingenz
} 




Abb. 1: GEM in Verbindung mit einer Auswahl schulgeographischer Konzepte (eigener Entwurf)

Organisationskonzepte die hierarchischen Konzepte space, place und time $\mathrm{zu}$ organisieren und zu untersuchen, indem bspw. betrachtet wird, wie ein Ort repräsentiert ist (Organisationskonzept: perception and representation). Uhlenwinkel (2013a) formulierte Konzepte, die zum Großteil mit denen Taylors übereinstimmen: place, space, Maßstab, Wandel, Vernetzung, Diversität, Wahrnehmung und Darstellung.

Betrachtet man die Auswahl der Konzepte von Holloway et al. (2003), des britischen Curriculums (2007), Taylor (2008), Lambert und Morgan (2010), des australischen Curriculums (2014) und Uhlenwinkel (2013a) (siehe Abb. 1) fällt auf, dass, bis auf Taylor, alle place, space und scale als Kernkonzepte formulieren. Laut Uhlenwinkel (2013b) bleibt bei Taylor durch das Fehlen von scale allerdings eine geographisch zentrale Perspektive unbeachtet.

Es kann zu dem Schluss gekommen werden, dass Basiskonzepte in der Geographie-Lehramtsausbildung und im nationalen Lehrplan zum Großteil als Analysewerkzeug diskutiert und immer stärker verankert werden. Allerdings besteht keine einheitliche Anwendung der Basiskonzepte.

\subsection{Zusammenfassung und Ausgangsposition}

Folgende zentralen Positionen lassen sich zusammenfassen:

- Durch die definierten Ziele und Inhalte, die in der Schulgeographie behandelt werden, und die Art und Weise, wie die Inhalte für den Unter- richt aufbereitet werden, hat die Schulgeographie ein klar erkennbares Objekt, das sich im Ansatz der Wechselwirkungen zwischen Mensch und Umwelt zeigt. Die Ziele und Merkmale der Schulgeographie spiegeln sich jedoch in der Hochschulbildung nicht ausreichend wider. Es fehlt eine solide Grundlage für die Anforderungen auf Schulniveau.

- Die zu behandelnden Fachgegenstände für den Unterricht sind durch eine hohe Komplexität gekennzeichnet, welche zu berücksichtigen ist und welche unter Einbezug der Mensch-UmweltSchnittstelle erfolgen sollte, um Inhalte fachlich und angemessen im Unterricht zu vermitteln.

- Geographische Basiskonzepte werden als ein sinnvolles Analysewerkzeug von Inhalten für Lehrkräfte diskutiert.

Die zentrale Aufgabe besteht in der Professionalisierung von Geographielehramtsstudierenden, welche die Fähigkeit beinhaltet, das Fach zu reflektieren und Wissen zu dekonstruieren. Studierende sollten in der Lage sein, Komplexität zu erkennen und mit dieser umzugehen - ohne dabei zu übervereinfachen. Um diese Herausforderung anzugehen, bedarf es eines fachdidaktischen Instruments mit praktischer Anwendbarkeit, das hilft, die Struktur eines Problems und einer Situation unter geographischem Gesichtspunkt zu erschließen (Peter \& Nauss 2020). Im weiteren Verlauf wird die theoretische Herleitung dargelegt und das GEM erörtert. 




Abb. 2: Fachdidaktische Modellierung: verändert nach Peter und Nauss (2020)

\section{Eine geographische Perspektive zur Rekonstruktion des Fachverständnisses - mit Basiskonzepten}

Lehramtsstudierende müssen nicht über den Spezialisierungsgrad von Wissenschaftler*innen verfügen, müssen aber dennoch in der Lage sein, sich Fachwissen anzueignen und Strukturen und systemische $\mathrm{Zu}-$ sammenhänge zu erkennen. Eine Herausforderung besteht darin, fachwissenschaftliche Inhalte unter dem Vermittlungsaspekt zu reflektieren und für den Unterricht nutzbar zu machen. Ausgehend vom Dilemma zwischen Schulgeographie und Geographie auf Universitätsniveau und dem Fokus der Mensch-UmweltSchnittstelle in der Schule, sollte diese Schnittstelle integriert und bei der Professionalisierung angehender Lehrer*innen an der Universität explizit berücksichtigt werden.

Ein praxisnahes Anwendungskonzept ist an dieser Stelle erforderlich (siehe Abb. 2). Fachwissen soll rekonstruiert und entwickelt werden. Um eine zu starke inhaltliche Eingrenzung oder Übervereinfachung zu vermeiden, setzt $G E M$ vor der didaktischen Analyse an (siehe Abb. 3) und kann als Hinführung zur Sachanalyse genutzt werden. Das Konzept lässt sich im Modell der didaktischen Rekonstruktion (Kattmann 2007: 94) im Bereich der ,fachlichen Klärung' einordnen. Innerhalb des GEMs werden die Basiskonzepte place, space und scale als gleichwertige Hauptkonzepte betrachtet, welche wie auch bei anderen zuvor genannten Autor*innen Kernkonzepte darstellen (siehe Abb. 1). Es ist ein Ansatz, durch den das Konzept scale und die Dualität von space und place berücksichtigt werden soll. Auch im GEM werden die drei Konzepte als Basiskonzepte bezeichnet, da sie eine inhaltliche Strukturierungshilfe darstellen können, als ein Werkzeug für Studierende und Lehrkräfte genutzt werden sollen, geographisches Wissen und Verständnis (Brooks 2013) abbilden sollen und sie ferner ein didaktisches Ziel verfolgen. Andere Konzepte wie change und diversity etc. werden wegen (i) der Überschneidungen von Bedeutung und Inhalt (siehe unten) und (ii) des Fehlens fachspezifischer Fachgrenzen nicht berücksichtigt. Lambert (2013: 175) stellte bereits dar, dass die Konzepte ,place, space und Umwelt eine beständige Struktur darstellen [...], die andere Konzepte wie Wandel und Vernetzung beinhalten“. Je nach verfolgtem Ziel und Zweck entstehen unter Anwendung von $G E M$ eine oder mehrere Leitfragen, die andere Konzepte beinhalten bzw. während der Modellierungs- und Strukturierungsphase berücksichtigt werden können (siehe Kap. 5).

\section{Inhaltliche Strukturierung durch Model- lierung und Basiskonzepte - GEM}

Ausgehend davon, dass geographische Inhalte einer Komplexität unterliegen, soll hier eine Möglichkeit aufgezeigt werden, mit Komplexität umzugehen und sie zu dekonstruieren. In diesem Sinne kann GEM genutzt werden, um sich komplexe Systeme aus der Perspektive der Systemdynamik zu eröffnen, einen Zugang zu finden und leitfragenorientiert Fachgegenstände $\mathrm{zu}$ erschließen, indem die Konzepte space, place, scale genutzt werden. Durch eine naturwissenschaftliche und sozialwissenschaftliche Betrachtung werden die wesentlichen Inhalte und Zusammenhänge des Systems veranschaulicht (siehe Abb. 4/Tab. 1).

GEM umfasst dabei eine Modellierungsphase und eine anschließende Strukturierungsphase, in der ein Lehrgegenstand gebildet wird (siehe Abb. 3 \& Tab. 1), d.h. Fachinhalte werden in Lehrinhalte überführt. Die Lehrinhalte bestehen aus Einheiten, die Fragestellungen aus sowohl physisch-räumlichen als auch sozialräumlichen Perspektiven betrachten können. Bezogen auf das „doppelte Praxisverständnis“ entspricht der Modellierungsprozess der ersten Praxis und der Strukturierungsprozess mit der fachdidaktischen Erschlie- 
ßung dem Übergang zur zweiten Praxis, in der die Unterrichtsplanung verortet ist. Für unseren Zweck haben wir diese an das GEM anschließenden Phasen konkretisiert und in Unterrichtsplanung und Unterrichtsdurchführung unterteilt (siehe Abb. 3).

Voraussetzung für die Strukturierung ist die Konstruktion von Modellen als ,,key tools for scientists, science teachers and science learners" (Coll et al. 2005: 183). Dem Kompetenzmodell von Ammoneit et al. (2019) folgend spielt die räumliche Herangehensweise beim Modellieren eine entscheidende Rolle, um die Komplexität geographischer Inhalte zu dekonstruieren. GEM orientiert sich an der Komplexitätstheorie von Luhmann (vgl. 1995), die eine Komplexitätsreduktion durch Modellierung von Systemen ermöglicht. So wird zur Komplexitätsdekonstruktion ein System autopoietisch generiert. Es schafft sich selbst seine Grenzen. Dabei kann System auch als eine soziale Einheit betrachtet werden, als ,,complexes of elements in interaction to which certain systems laws can be applied“ (Bertalanffy 1951: 307), deren Komplexität mit der Anzahl der Elemente und Beziehungen, genauer der Anzahl der Rückkopplungen, zunimmt. Nach Ammoneit et al. (2019) ergibt sich bei der geographischen Modellierung dabei die Funktion aus der Komposition und Konfiguration der räumlich angeordneten Elemente.

Für unseren Zweck wird ein System in Bezug auf den Fachgegenstand modelliert. Dabei muss das System nicht die reale Welt darstellen. In diesem Sinne postuliert bereits Luhmann (1995), der generellen Systemdynamik von z. B. Forrester (1985) folgend, dass Systeme von ihrer Umwelt abzugrenzen sind. Die Umwelt stellt dabei die Randbedingungen, unter denen das System betrachtet wird und trennt damit Umwelt als das, was implizit berücksichtigt wird von Elementen und Beziehungen, die explizit in der Systembetrachtung berücksichtigt werden. Es muss stets gefragt werden: Was ist explizit und was ist implizit im Systemmodell zu berücksichtigen? Auf diese Weise kann Komplexität dekonstruiert werden. Nicht-Systeme wie Welt oder Umwelt sind dabei immer implizit zu betrachten. Die These, dass sich etwas, das als Element funktioniert, nicht ohne System charakterisieren lässt und dass organisierte Komplexität nur durch die Bildung von Systemen erfasst werden kann, macht dies deutlich (vgl. Luhmann 1991).

Wie auch im Mensch-Umwelt-System der DGfG (2020) können Räume als Systeme betrachtet werden. Ein real existierender Raum ist in allen Details und Beziehungen kaum erfassbar, so dass ein System in dem GEM funktional abgegrenzt, also modelliert werden muss. Systeme wie Ökosystem, Innovationssystem etc. sind damit das Ergebnis einer Modellierungsaktivität. Am Beispiel des Brandrodungswan- derfeldbaus (siehe. Abb. 4) wird die Rolle des Systems mit seinen funktionalen Elementen und Beziehungen unter verschiedenen physischen und sozialen Perspektiven veranschaulicht. Durch die jeweils unterschiedliche Systemabgrenzung werden jeweils konstruierte Raumausschnitte definiert, die explizit betrachtet werden.

\subsection{Space und Place als Zustandsgrößen des Systems}

Space und place gehören zu den am häufigsten genannten geographischen Konzepten in der Literatur (vgl. Clifford et al. 2009; Jackson 2006; Lambert \& Morgen 2010; Taylor 2008, 2011), (siehe Abb. 1). Für place gibt es keine einheitliche Definition (vgl. Lambert \& Morgan 2010). Unterschiedliche places werden basierend auf einen bestimmten Zweck, den sie als Ort erfüllen, benannt (z. B. Bars, Museen, Straßen, Parks usw.). Sie sind wie Cresswell (2015: 12) formuliert „a meaningful location” oder spezifischer ,the notion of a meaningful segment of geographical space" (2008: 132). Ihnen wird eine soziale, subjektive oder gruppenspezifische Bedeutung zugemessen. Massey (1997: 322) weist darauf hin, dass place konstruiert ist ,out of a particular constellation of social relations, meeting and weaving together at a particular locus". Sie sind jedoch nicht unveränderlich (vgl. Cresswell 2008).

Space wird von Taylor (2005: 14) als ,,the physical dimension in which things exist, while place is space given meaning" definiert. Dadurch ist place ein Teil von space und entsteht im space (vgl. Taylor 2005). Freytag (2014) definiert space als abstrakt und geometrisch und dass dieses Konzept Menschen und Objekte und deren Beziehungen an bestimmten Orten enthält. Tuan (1977) versteht space als Bewegung, welche ermöglicht, jeden Ort in einem space als place zu definieren.

Space und place in dem GEM definieren die Systemgrenze, also den Ausschnitt der Betrachtungsgrenze. Fachgegenstände können damit durch einen naturwissenschaftlichen oder sozialwissenschaftlichen $\mathrm{Zu}$ gang zum System erschlossen werden (siehe Abb. 4). Nach unserem Verständnis können places und spaces als Zustandsgrößen, als eine Systemkomponente verstanden werden. Sie sind somit Bestandteil des Systems und werden durch den fachlichen Zugang rein funktional ausgewählt.

Funktionen und Wechselwirkungen zwischen den places und spaces können als Pfeile oder Linien dargestellt werden. Darüber hinaus stellt das Verständnis von place und space nicht unbedingt Orte an einem bestimmten Punkt auf der Erdoberfläche dar. Es bedarf keiner objektiven Koordinaten, um sie als Raum zu 
definieren. Space ist analog zu Kant (1781) vielmehr die Raumperspektive, unter der man Objekte wahrnimmt. Space wird damit als das funktional definierte Fenster unseres Systems verstanden, das man explizit betrachtet. Ein space kann bspw. ein bewirtschaftetes Feld, ein Sektor eines Primär- oder Sekundärwaldes, ein einzelner Baum, eine indigene Siedlung im Tropischen Regenwald oder der Ausschnitt eines Satellitenbilds sein (siehe Abb. 4). Wie auch bei anderen Autor*innen (vgl. Cresswell 2015; Massey 1997; Taylor 2005, 2008) wird durch Anwendung des GEMs einem place eine soziale Bedeutung zugemessen, indem man ihm einen Grund für die Existenz gibt. Place wird verstanden als ein Raum, der im Handeln von Menschen unterschiedlich wahrgenommen wird. Demnach wird place durch eine sozial-geographische Perspektive erschlossen und modelliert (siehe Abb. 4 \& Tab. 1).

Unser funktional bestimmtes System kann Objekte, Menschen, physische und soziale Prozesse oder eine Kombination dieser Inhalte zu einem bestimmten Zeitpunkt enthalten. Innerhalb der modellierten Prozessgrenzen können Zusammenhänge wie MenschUmwelt-Systeme und Phänomene beobachtet und analysiert werden. Die Grenze, die ein space oder place definiert, verhindert, dass eine aus dem System resultierende Antwort wieder in das System wechselt. Es ist generell verboten, dass Rückkopplungen solchen Systemen trotzen; sonst könnte Komplexität nicht reduziert werden. Die System-Umwelt-Grenze ist damit auch die Grenze für Rückkopplungen, die noch explizit betrachtet werden.

\subsection{Scale als Hilfsmittel zur Definition von Systemgrenzen}

Indem man das Konzept scale (siehe anschließenden Abschnitt) verwendet, verändert man das Auflösungsniveau und den Blickwinkel innerhalb eines space und den darin explizit betrachteten places. Zoomt man z. B. von einem funktionellen Teil des Regenwaldes (space), wo Brandrodungswanderfeldbau betrieben wird (siehe Abb. 4: Bild 1), in ein einzelnes Feld (neuer space) (siehe Abb. 4: Bild 1a), verändert man den Blickwinkel und kann betrachten, was auf diesem Feld angebaut wird. Das Konzept scale wird demnach dazu benötigt, einzelne Phänomene oder Prozesse genauer zu betrachten und die Systemgrenzen neu zu definieren. Der ausgewählte Betrachtungsbereich entscheidet darüber, welche Komponenten und Funktionen explizit und welche implizit als Randbedingung beachtet werden (vgl. Peter \& Nauss 2020). Durch diese Anwendung des Konzepts scale kann dieses in Form einer geographischen Skala verstanden werden, die das räumliche Ausmaß eines Phänomens beschreibt (Lambert \& Morgan 2010: 98) und eine naturwissenschaftliche und sozialwissenschaftliche Betrachtung ermöglicht.

\subsection{Time als implizite Modellkomponente}

Für das GEM spielen neben räumlichen Aspekten auch zeitliche eine entscheidende Rolle. Das Basiskonzept time wird nicht als separates Konzept



Abb. 3: Modellierung und Strukturierung einer Situation und eines Problems im Hinblick auf die Unterrichtsplanung und Durchführung mithilfe des GEMs (eigener Entwurf) 




Abb. 4: Modellierung des Brandrodungswanderfeldbaus, Problembeispiel: Bevölkerungsdruck, Raubbau im Tropischen Regenwald (eigener Entwurf)

gesehen, sondern als inhärenter Bestandteil der Systemmodellierung. D.h., dass die Modellierung eines space und place immer den Aspekt der Zeit beinhaltet, weil spaces und places nicht stillstehen. Massey stellt dies dar, indem sie sagt:,,[s]pace has time/times within it. This is not the static simultaneity of a closed system but a simultaneity of movements" (Massey 2003: 108). Brooks und Morgen (2006) weisen ferner darauf hin, dass wenn places untersucht werden, immer ein bestimmter Zeitpunkt und auch historische Einflüsse berücksichtigt werden. Das GEM berücksichtigt demnach immer die zeitliche Komponente während der Modellierungsprozesse.

Nachfolgend soll mithilfe des GEMs ein Problem geographisch erschlossen und das Vorgehen beschrieben werden.

\section{Anwendung des Geographischen Er- schließungsmodels - Ein Beispiel}

Die Modellierung von Lehrgegenständen wird in diesem Abschnitt konkretisiert und anhand des Brandrodungswanderfeldbaus (Afrika/Südamerika), Problembeispiel: Bevölkerungsdruck/Raubbau im Tropischen Regenwald verdeutlicht (vgl. Hessisches Kultusministerium 2010, hessischer Lehrplan für die 8. Klasse [G9] des Gymnasialzweigs).

\subsection{Modellierungsphase}

Die Modellierungsphase umfasst zwei Schritte:

(1) Eine Situation und ein Problem definieren und eine Frage formulieren

(2) Erschließung anhand eines fachlichen Zugangs mithilfe der Konzepte space, place und scale

Im Gegensatz zu Taylors Ansatz (vgl. 2011) entstehen die Fragen nicht aus den Basiskonzepten, sondern mit Blick auf das räumliche Problem des Brandrodungswanderfeldbaus im Kontext von Bevölkerungsdruck und Raubbau. Die Fragestellung ist: Warum führen der Brandrodungswanderfeldbau und der Bevölkerungsdruck zu Übernutzung im Tropischen Regenwald? Die Strukturierung wird durch die Betrachtung eines Raumausschnitts vorgenommen. Es wird ein funktionell abgegrenztes System konstruiert, das gleichzeitig die Raumbegrenzung und das Untersuchungsgebiet darstellt. Um den Modellierungsvorgang besser nachvollziehbar zu machen, wurden den spaces und places Namen gegeben.

\subsubsection{Traditioneller Brandrodungswander feldbau (1)}

Das System (siehe Abb. 4: Bild 1) besteht aus einem space und einem place. Darin befindet sich eine Siedlung, indigene Völker und Felder, die durch den Prozess der Brandrodung entstanden sind. In der Rand- 
bedingung (Umgebung) befinden sich Elemente wie Tropischer Regenwald, Klima und Bodenbeschaffenheit, die als gegeben angesehen werden. Anschließend kann man sich explizit auf die physische Betrachtungsweise des Habitats im space und somit den Prozess der Brandrodung konzentrieren.

Durch eine soziale Betrachtungsweise des Brandrodungswanderfeldbaus mithilfe des Konzepts place wird die Tradition der dort ansässigen Bevölkerung deutlich und bspw. der Nutzen, die Einstellung und die persönliche Begründung der Handlung (siehe Abb. 4: Bild 1). Die Brandrodung (soziale Handlung) hat physische Auswirkungen auf den Tropischen Regenwald und der Boden benötigt Regenerationszeit. Was jedoch auf den Feldern passiert (Art der Kulturen, Anbauzeit etc.), wird zunächst wie eine Blackbox behandelt.

\subsubsection{Anbaukulturen (1a)}

Im Folgenden kann eines oder mehrere Felder physisch als space betrachtet werden, (siehe Abb. 4: Bild 1a). Hierbei nutzt man scale ausgehend von Bild 1 und modelliert ein weiteres System mit einem space, welches als physischer Raum ein Feld innerhalb des Brandrodungswanderfeldbaus darstellt. Hier sieht man dann die (verschiedenen) Anbaukulturen innerhalb des Feldes. Ein sozialer Zugang könnte über bspw. die Bedeutung der Feldfrucht für die einzelnen Bauern*Bäuerinnen erfolgen.

\subsubsection{Brandrodungswanderfeldbau und Nut- zungskonflikte (2)}

Auch Bevölkerungsdruck und Raubbau können einbezogen werden (siehe Abb. 4: Bild 2). Das modellierte und funktional abgegrenzte System würde mit seinem space die Dichte der Gesamtbevölkerung bspw. in Amazonien darstellen. Hier berücksichtigt man indigene Völker und den wachsenden Bevölkerungsdruck. Durch diese Perspektive wird deutlich, dass indigene Völker nicht mehr in der Lage sein werden, ein neues Gebiet im Tropischen Regenwald zu roden, sondern früher als im traditionellen Turnus in ein in der Vergangenheit aufgegebenes Feld zurückkehren müssen. Das erste Ergebnis, d.h. die Systemreaktion auf den Treiber „Bevölkerungsdruck“ ist, dass Menschen eine Lösung durch Beschleunigung finden: eine frühe Rückkehr auf zuvor bewirtschaftete Felder oder eine Veränderung des Anbaus. Der soziale Zugang könnte dann durch einen place, welcher die Einstellung und Meinung der dort ansässigen Bevölkerung in Bezug auf die räumliche Enge thematisiert, modelliert werden (siehe Abb. 4: Bild 2).

\subsubsection{Bevölkerungswachstum und Raubbau (}

Möchte man den Bevölkerungsdruck und Raubbau untersuchen, kann man ein System mit einem space und mehreren places modellieren (siehe Abb. 4: Bild 3).

Betrachtet man den space, kommen hier Aspekte wie die verstärkte Übernutzung der Felder durch andere Siedlungen und (globale) Unternehmen zum Tragen. Dieser Prozess könnte durch ein früheres Bevölkerungswachstum in den umliegenden Städten begleitet werden (Randbedingung: Bevölkerungswachstum). Ein Resultat daraus kann die Erschließung neuer Anbauflächen sein, um die wachsende Bevölkerung mit Nahrung zu versorgen.

Als einen place könnte man die Stadtregierung als Interessengruppe darstellen, welche in diesem Zusammenhang mit Infrastrukturmaßnahmen wie dem Bau von Straßen, um den Regenwald zugänglich zu machen, und Immobilienprojekten, die den indigenen Völkern Wohnungen am Stadtrand anbieten, reagieren (Randbedingung: Entwicklung des Regenwaldes) (siehe Abb. 4: Bild 3: Place 1).

Ein weiterer Aspekt könnte die Ansiedlung von (globalen) Unternehmen sein, die aus wirtschaftlichen Entscheidungen, Konsumverhalten oder aus Gründen des globalen Handels entstehen (place 2). Die Systemreaktion auf diese Perspektive des Problems wäre, dass der Bevölkerungsdruck und die Unternehmen die Übernutzung verstärken (siehe Abb. 4: Bild 3).

Die indigenen Völker fühlen sich dadurch möglicherweise gezwungen, ihren Standort zu verlassen (place 3) (siehe Abb. 4: Bild 3).

Aufgrund der vorherrschenden natürlichen Bedingungen im Tropischen Regenwald betreibt ein Teil der ansässigen Bevölkerung Brandrodungswanderfeldbau. Der Bevölkerungsdruck übt Einfluss auf das menschliche Handeln vor Ort aus, was das traditionelle Handeln ins Ungleichgewicht bringen könnte. Das daraus resultierende Handeln der indigenen Völker kann bspw. Auswirkungen auf die Umwelt haben. So weist der Sekundärwald eine geringere Artenvielfalt auf als der Regenwald und die Übernutzung hat einen negativen Einfluss auf die Natur (Verlust der Biodiversität, Bodenerosion etc.).

Die Beziehung der Mensch-Umwelt-Schnittstelle kann durch die modellierten spaces und places herausgearbeitet und verdeutlicht werden. Zudem strukturieren sie den Lehrgegenstand in Lehrinhalte und sind die Grundlage der Unterrichtsplanung (siehe Tab. 1).

Basiskonzepte wie change, interaction, interdependencies finden sich in dem GEM innerhalb des Systems wieder. Bei Betrachtung der Beziehungen unter den spaces und places wird dies deutlich, bspw. durch den zunehmenden Bevölkerungsdruck oder die Verände- 
rung des Bodens durch die Bewirtschaftung, die den Wanderfeldbau charakterisiert.

\subsection{Strukturierungsphase}

Der Strukturierungsprozess formuliert den Lehrgegenstand eines bestimmten modellierten Systems mit seinen spaces und places und führt zu spezifischen inhaltlichen Zielen, die anschließend wiederum als Unterrichtsziele für die Planung der Unterrichtseinheit umgesetzt werden können. Für diesen Prozess müssen die angehenden Lehrpersonen die Ergebnisse des Erschließungsprozesses einbeziehen. Darüber hinaus werden die curricularen Richtlinien berücksichtigt.

Strukturierte Lehrgegenstände zum Brandrodungswanderfeldbau (Afrika/Südamerika), Problembeispiel: Bevölkerungsdruck/Raubbau im Tropischen Regenwald sind in der folgenden Tabelle beispielhaft zu sehen.

Die strukturierten Lehrinhalte sollen bei diesem Prozess helfen, inhaltliche Aspekte zu finden, die für einen geographischen Ansatz, den Zugang zum jeweiligen Ziel und für das Verständnis des Problems erforderlich sind. Der Lehrgegenstand stellt das Problem innerhalb eines modellierten Systems als Einheiten dar. Dieses Vorgehen hilft bei der Entscheidung, welche spezifischen Inhalte für das Verständnis und die Vermittlung des Fachgegenstands notwendig sind (siehe Tab. 1). Wie auch in dem 2007 National Curriculum für GB (siehe Kap. 2.3) werden Inhalte gruppiert. In dem GEM befinden sich die gruppierten Inhalte in den modellierten spaces und places und müssen nun in der Strukturierungsphase in Lehrgegenstände mit zugehörigen Inhalten gewandelt werden (siehe Tab. 1). Dieser Prozess legt grobe Ziele des Problems in Form von Inhalten fest, die in der Unterrichtseinheit vermittelt werden sollen. Zudem kann ein Rückbezug zur Fragestellung hergestellt werden.

Nach der Strukturierung des Lehrgegenstandes kann mit der Planung der Unterrichtseinheit/-stunde begonnen werden. Diese Phase wird als Unterrichtsplanung bezeichnet. Hier werden die Bildungsziele formuliert, die Vorkenntnisse der Schüler*innen und Inhalte, die für den Unterricht benötigt werden berücksichtigt, um die Stunde bzw. Einheit zu planen. Die didaktische Reduktion und didaktische Analyse der Inhalte geschieht dann basierend auf Lerngruppe und Jahrgangsstufe.

Tab. 1: Mögliche Strukturierung des Lehrgegenstandes gemäß. Abb. 4 (eigener Entwurf)

\section{Bild}

Bild 1 Nachhaltiger (traditioneller) Brandrodungswanderfeldbau

Bild la Die Bedeutung der Regenerationszeit des Brandrodungswanderfeldbaus im Tropischen Regenwald

Bild 2 Brandrodung und räumliche Nutzungskonflikte im Tropischen Regenwald

Bild 3 Bevölkerungswachstum, Megacitys und (globale) Unternehmen erhöhen zunehmend den Druck im Kontext des Brandrodungswanderfeldbaus

- Überbeanspruchung/ Raubbau im Tropischen Regenwald

\section{Lehrinhalt}

- space: Indigene Völker brandroden Felder, die durch den Prozess des Brandrodungswanderfeldbaus im Umfeld der Dörfer entstanden sind.

- place: Einstellung und Meinung der ansässigen Bevölkerung zum Brandrodungswanderfeldbau, Begründung der Handlung.

- space: Beobachtung eines Feldes, primärer Wald - kurzgeschlossener Nährstoffkreislauf (und sekundärer Wald - Bild lb).

Nachhaltige Landwirtschaft braucht Regenerationszeit im Tropischen Regenwald.

- space: Dichte der Gesamtbevölkerung im definierten Raum (Bevölkerungswachstum).

Limitierte Fläche/Raum (im Kontext von Zeit/time mit hohem Bevölkerungsdruck) führt bei indigenen Völkern, die Brandrodungswanderfeldbau betreiben, zu einer früheren Rückkehr auf ein zuvor bewirtschaftetes Feld oder Veränderung des Anbaus (Landnutzungskonflikte).

- place: Einstellung und Meinung der ansässigen Bevölkerung in Bezug auf die räumliche Enge.

- space: Die Bevölkerung wächst $\rightarrow$ die Nachfrage nach Agrarprodukten steigt $\rightarrow$ mangelnde Regenerationsperiode führt zur Übernutzung $\rightarrow$ (globale) Unternehmen verschärfen das Problem des Landnutzungskonflikts.

- place 1: Interessengruppe: Stadtregierung Immobilienprojekte, Infrastrukturmaßnahmen etc.

- place 2: Interessengruppe: (globale) Unternehmen wirtschaftliche Entscheidungen etc.

- place 3: Interessengruppe: indigene Völker wollen bspw. ihren Standort nicht verlassen.

\section{Schlussfolgerungen und Ausblick}

Zusammenfassend lässt sich sagen, dass sich die Schulgeographie insbesondere mit Mensch-Umwelt-Beziehungen beschäftigt. Obwohl diese in der universitären Lehramtsbildung oftmals nicht als ein Hauptbestandteil der fachwissenschaftlichen Lehre zu finden sind, fordert man es von den Studierenden in Hinblick auf den Schulalltag. Verschärft wird die Problematik dahingehend, dass (curriculare) Mensch-Umwelt-Themen hoch komplexe Systeme darstellen. Das GEM liefert hier die Brückenfunktion zwischen der physischen Geographie und der Humangeographie zur Erschließung der Fachgegenstände. Der Nutzen des GEMs liegt darin, dass Studierende und Lehrpersonen durch ein besseres natur- und sozialwissenschaftliches Verständnis von Fachgegenständen in die Lage versetzt werden, Geographieunterricht fachlich fundierter zu planen und zu unterrichten. Der Modellierungs- und Strukturierungsprozess durch die Anwendung von GEM könnte insbesondere die 
Analyse von für Bildung für nachhaltige Entwicklung relevante Thematiken unterstützen. In diesem Bereich sind weitere Überlegungen möglich.

Das GEM muss im Hinblick auf Praktikabilität und Nutzen empirisch untersucht werden. Eine Anwendung in allen Phasen der Lehrkräfteausbildung ist denkbar.

\section{Dank}

Die Publikation dieses Beitrags wurde aus den Mitteln des Open-Access Publikationsfonds der Universität Marburg unterstützt.

\section{$7 \quad$ Literatur}

acara Australian curriculum (2014): assessment and reporting authority. 7-10 Geography: subject [rationale]. https://www.australiancurriculum.edu.au/f-10-curriculum/humanities-and-social-sciences/geography/rationale/ (12.06.2019)

Ammoneit, R., C. Reudenbach, A. Turek, T. Nauss \& C. Peter (2019): Geographische Modellierkompetenz - Modellierung von Raum konzeptualisieren. In: GW-Unterricht 156. S. 19-29.

Arslan, H. O., C. Cigdemoglu \& C. Moseley (2012): A Three-Tier Diagnostic Test to Assess Pre-Service Teachers' Misconceptions about Global Warming, Greenhouse Effect, Ozone Layer Depletion, and Acid Rain. In: International Journal of Science Education 34(11). S. 1667-1686.

Bertalanffy, L. (1951): Problems of general system theory. In: Human Biology 23. S. 302-312.

Brooks, C. (2013): How do we understand conceptual development in school geography? In: Lambert, D. \& M. Jones (Eds.): Debates in subject teaching series. Debates in geography education. Milton Park, Abingdon, Oxon. Routledge, New York. S. 75-88.

Brooks, C. \& A. Morgan (2006): Theory into practice. Cases and places. Geographical Ass, Sheffield.

Clifford, N. J. (2002): The future of Geography: when the whole is less than the sum of its parts. In: Geoforum 33(4). S. 431-436.

Clifford, N. J., S. L. Holloway, S. Rice \& G. Valentine (2009): Key concepts in geography. 2. Aufl. SAGE, Los Angeles.

Coll, R. K., B. France \& I. Taylor (2005): The role of models/and analogies in science education: implications from research. In: International Journal of Science Education 27(2). S. 183-198. http://www.tandfonline.com/ doi/abs/10.1080/0950069042000276712 (12.06.2019)

Cresswell, T. (2008): Place: encountering geography as philosophy. In: Geography 93(3). S. 132-139.

Cresswell, T. (2015): Short introductions to geography. Place: A short introduction ([Repr.]). Blackwell, Malden, Mass.

DGfG. (2020): Bildungsstandards im Fach Geographie für den Mittleren Schulabschluss mit Aufgabenbeispielen. Bonn.
Fögele, J. (2015): Mit geographischen Basiskonzepten Komplexität bearbeiten. Hintergrund und Anwendung am Beispiel der Ressource ,,Sand“. In: Geographie aktuell \& Schule 37(216). S. 11-21.

Fögele, J. (2016): Entwicklung basiskonzeptionellen Verständnisses in geographischen Lehrerfortbildungen: Rekonstruktive Typenbildung | Relationale Prozessanalyse Responsive Evaluation. Geographiedidaktische Forschungen 61. Monsenstein und Vannerdat, Münster.

Forrester, J. W. (1985): Memo / Industrial dynamics research: D-16: Formulating quantitative models of dynamic behavior of industrial and economic systems. Massachusetts Institute of Technology, School of Industrial Management. Cambridge, Mass.

Freytag, T. (2014): Raum und Gesellschaft. In: Lossau, J., T. Freytag \& R. Lippuner (Hrsg.): UTB Geographie, Sozialwissenschaften, Kulturwissenschaften 3898. Schlüsselbegriffe der Kultur- und Sozialgeographie. Eugen Ulmer, Stuttgart. S. 12-24.

Geist, H. J. (2018): Integrative Geographie neu denken z. B. anthropozänisch. In: Geographica Helvetica 73(2). S. $187-191$.

Heffron, S. G. \& R. M. Downs (2012): Geography for life: National geography standards. 2. Aufl. National Council for Geographic Education, Washington, DC.

Hemmer, I. \& M. Hemmer (2000): Qualität der Lehrerausbildung im Fach Geographie aus Sicht der Fachleiter/Seminarleiter: Ergebnisse einer deutschlandweiten Befragung. In: Geographie und ihre Didaktik 2. S. 61-87.

Hemmer, I. \& G. Obermeier (2003): Qualität der Lehrerbildung an der Universität - Lehrerbefragung zur Ausbildung in Geographie, Geographiedidaktik und in den Erziehungswissenschaften in Bayern. In: Geographie und ihre Didaktik 2. S. 80-109.

Hericks, U., N. Meister \& W. Meseth (2018): Professionalisierung durch Perspektivwechsel? Lehramtsstudierende zwischen schulischer und universitärer Praxis. In: Artmann, M., M. Berendonck, P. Herzmann \& A. Liegmann (Hrsg.): Professionalisierung im Praxissemester. Beiträge qualitativer Forschung aus Bildungswissenschaft und Fachdidaktik zu Praxisphasen in der Lehrerbildung. Bad Heilbrunn.

Hericks, U. \& R. Laging (2020): Wie man im Fachlichen professionell wird? Reflexionen zur Lehrerbildung. In: Heer M. \& U. Heinen (Hrsg.): Die Stimmen der Fächer hören. Fachprofil und Bildungsanspruch in der Lehrerbildung. Ferdinand Schöningh, Paderborn. S. 117-136.

Hericks, U. \& N. Meister (2020): Das Fach im Lehramtsstudium - theoretische und konzeptionelle Perspektiven. Einführung in den Band. In: Meister, N., U. Hericks, R. Kreyer \& R. Laging (i. E.) (Hrsg.): Zur Sache. Die Rolle des Fachs in der universitären Lehrerbildung. Springer Verlag, Wiesbaden.

Hessisches Kultusministerium (2010): Lehrplan Erdkunde. Gymnasialer Bildungsgang. Jahrgangsstufen 5 bis 13. https://kultusministerium.hessen.de/sites/default/files/ media/g9-erdkunde.pdf (22.10.2019) 
Jekel, T. \& H. Pichler (2017): Vom GW-Unterrichten zum Unterrichten mit geographischen und ökonomischen Konzepten. In: GW-Unterricht 147. S. 5-15.

Jackson, P. (2006): Thinking Geographically. In: Geography 91(3). S. 199-204.

Kant, I. (1781): Kritik der reinen Vernunft. 1. Aufl. „Der transzendentalen Ästhetik, Erster Abschnitt, Von dem Raume“, „Der Transzendentalen Analytik, Zweites Hauptstück, Von der Deduktion der reinen Verstandesbegriffe“. Bd. 3. S. 71-77.

Kattmann, U. (2007): Didaktische Rekonstruktion - eine praktische Theorie. In: Krüger, D. \& H. Vogt (Hrsg.): Theorien in der biologischen Forschung. Springer Verlag, Wiesbaden. S. 93-104.

Khalid, T. (2003): Pre-service high school teachers' perceptions of three environmental phenomena. In: Environmental Education Research 9(1). S. 35-50.

KMK/Konferenz der Kultusminister der Länder in der Bundesrepublik Deutschland (2004): Bildungsstandards im Fach Biologie für den mittleren Schulabschluss. Beschluss der Kultusministerkonferenz vom 16.12.2004. Bonn. http://db2.nibis.de/1db/cuvo/datei/bs_ms_kmk_ biologie.pdf (08.08.2019)

Krauss, S., M. Neubrand, W. Blum, J. Baumert, M. Brunner, M. Kunter \& A. Jordan (2008): Die Untersuchung des professionellen Wissens deutscher Mathematik-Lehrerinnen und -Lehrer im Rahmen der COACTIV-Studie. In: Journal für Mathematikdidaktik (JMD) 29(3/4). S. 223-258.

Kunter, M., J. Baumert, W. Blum, U. Klusmann, S. Krauss \& M. Neubrand (2011): Professionelle Kompetenz von Lehrkräften - Ergebnisse des Forschungsprogramms COACTIV. Waxmann, Münster.

Lambert, D. (2007): Connected lives: the importance of geographical perspectives. In: The Development Education Journal 13(3). S. 13-15.

Lambert, D. \& J. Morgan (2010): Teaching geography, 11-18. Routledge, London.

Lambert, D. (2013): Geographical concepts. In: Rolfes, M. \& A. Uhlenwinkel (Hrsg.): Metzler Handbuch 2.0 Geographieunterricht: Ein Leitfaden für Praxis und Ausbildung. Westermann, Braunschweig. S. 174-181.

Luhmann, N. (1991): Suhrkamp-Taschenbuch Wissenschaft: Vol. 666. Soziale Systeme: Grundriss einer allgemeinen Theorie. 4. Aufl. Suhrkamp, Frankfurt am Main.

Luhmann, N. (1995): Gesellschaftsstruktur und Semantik: Studien zur Wissenssoziologie der modernen Gesellschaft: Vol. 4. Suhrkamp, Frankfurt am Main.

Massey, D. (1997): A global sense of place. In: Barnes, T. \& D. Gregory (Eds.): Reading human geography: The poetics and politics of inquiry. Arnold, London.

Massey, D. (1999): Philosophy and politics of spatiality: some considerations: The Hettner-Lecture in Human Geography. In: Geographische Zeitschrift 87. S. 1-12.

Massey, D. (2003). Some Times of Space. In: Olafur, E. (Hg.): The Weather Project. Exhibition catalogue. Tate
Publishing, London. S. 107-118. http://www.f-i-e-l-d. co.uk/writings-violence_files/Some_times_of_space.pdf (28.05.2020)

National curriculum in England, Department of Education, GOV.UK (2013): Statutory guidance: National curriculum in England: geography programmes of study. https://www.gov.uk/government/publications/national-curriculum-in-england-geography-programmes-ofstudy/national-curriculum-in-england-geography-programmes-of-study (12.06.2019)

National Research Council (2012): A Framework for K-12 Science Education: Practices, Crosscutting Concepts, and Core Ideas (Committee on a Conceptual Framework for New K-12 Science Education Standards. Board on, Trans.). The National Academies Press, Washington, DC.

Neuweg, G. H. (2014): Das Wissen der Wissensvermittler. Problemstellungen, Befunde und Perspektiven der Forschung zum Lehrerwissen. In: Terhart, E., H. Bennewitz \& M. Rothland (Hrsg.): Handbuch der Forschung zum Lehrerberuf (2., vollst. überarb. u. erw. Aufl.). Waxmann, Münster. S. 583-614.

Ohl, U. (2013): Komplexität und Kontroversität. Herausforderungen des Geographieunterrichts mit hohem Bildungswert. In: Praxis Geographie 43(3). S. 4-8.

Peter, C. \& T. Nauss (2020): Der Raum im System. Von der geographischen Fachlichkeit zur modellierten Welt (-erschließung). In: Meister, N., U. Hericks, R. Kreyer \& L. Laging (Hrsg.): Zur Sache. Die Rolle des Faches in der universitären Lehrerbildung. Springer, Wiesbaden. S. 101-115.

Rawling, C. (2007): Theory into practice: professional development for geography teachers. Understanding place as a process. Geographical Association, Sheffield.

Reinfried, S. (2006): Conceptual Change in Physical Geography and Environmental Sciences through Mental Model Building. In: International Research in Geographical and Environmental Education 15(1). S. 41-61.

Shulman, L. S. (2004): The wisdom of practice: Essays on teaching, learning, and learning to teach. Wilson, S. (Ed.). Jossey-Bass Inc., San Francisco.

Steinbrick, M. \& P. Aufenvenne (2016): Integrative Geographiedidaktik? Versuch einer Positionsbestimmung der Fachdidaktik innerhalb der deutschsprachigen Geographie. In: GW-Unterricht 142. S. 5-17.

Taylor, L. (2005): Place: an exploration. In: Teaching Geography 30(1). S. 14-17.

Taylor, L. (2008): Key concepts and medium term planning. In: Teaching Geography 33(2). S. 50-54.

Taylor, L. (2011): Basiskonzepte im Geographieunterricht: Schlüssel, um die Welt besser zu verstehen und den Unterricht besser zu planen. In: Praxis Geographie 7/8. S. $8-15$.

Thrift, N. (2002): The future of geography. In: Geoforum 33. S. 291-298.

Tuan, Y.-F. (1977): Space and place: The perspective of experience. Univ. of Minnesota Press, Minneapolis, Minn. 
Uhlenwinkel, A. (2013a): Geographical concept: Place; Geographical concept: Space; Geographical concept: Maßstab; Geographical concept: Wandel; Geographical concept: Vernetzung. In: Rolfes, M. \& A. Uhlenwinkel (Hrsg.): Metzler Handbuch 2.0. Geographieunterricht: Ein Leitfaden für Praxis und Ausbildung. Westermann, Braunschweig. S. 182-216.

Uhlenwinkel, A. (2013b): Geographieunterricht im internationalen Vergleich. In: Kanwischer, D. (Hg.): Geographiedidaktik. Ein Arbeitsbuch zur Gestaltung des Geographieunterrrichts. Studienbücher der Geographie. Gebrüder Borntraeger Verlagsbuchhandlung, Stuttgart. S. 231-246.

Uphues, R. (2013): Basiskonzepte. In: Obermaier, G. \& D. Böhn (Hrsg.): Didaktische Impulse. Wörterbuch Geographiedidaktik. Begriffe von A-Z. Westermann, Braunschweig. S. 22-23.
Wardenga, U. (2011): Geographie als Brückenfach - oder: Arbeit am Mythos. Entgrenzt. In: Studentische Zeitschrift 1. S. 5-16.

Weichhart, P. (1999): Die Räume zwischen den Welten und die Welt der Räume: Zur Konzeption eines Schlüsselbegriffs der Geographie. In: Meusburger, P. (Hg.): Erdkundliches Wissen: Vol. 130. Handlungszentrierte Sozialgeographie: Benno Werlens Entwurf in kritischer Diskussion. Franz Steiner Verlag, Stuttgart. S. 67-94.

Weichhart, P. (2005): Auf der Suche nach der ,dritten Säule“. Gibt es Wege von der Rhetorik zur Pragmatik? In: Müller-Hahn, D. \& U. Wardenga (Hrsg.): Möglichkeiten und Grenzen integrativer Forschungsansätze in Physischer Geographie und Humangeographie. LeibnitzInstitut für Länderkunde, Leipzig. S. 109-136. 